\title{
Evolución del concepto de justicia con referencia al capitalismo y al socialismo
}

\author{
Dean Brackley, \\ Centro de Reflexión Teológica, \\ San Salvador.
}

La historia nos ofrece muchas definiciones de justicia. Siendo como es un concepto peligroso para todo siatus quo injusto, la justicia ha sido manejada y manipulada en todas las épocas. Por eso, aunque no fuese más que por esta razón, hay que volver a examinar en cada época lo que significa justicia, hay que desenmascarar los falsos conceptos que de ella se tienen.y, en cualquier caso, hay que historizar el concepto como pedía Ignacio Ellacuría.

En esce ensayo vamos a comenzar ofreciendo una primera definición de justicia, partiendo de la injusticia real (sección 1). Analizaremos después la historia del concepto en la Biblia (sección 2), en el pensamiento occidental (sección 3), en la uadición católica (sección 4) y en la ética latinoamericana de la liberación (sección 5), todo lo cual enriquecerá el concepto que esbozamos en la primera sección. Por úllimo, indicaremos cómo este concepto de justicia que hemos elaborado puede servir para evaluar los sistemas sociales del capitalismo y el socialismo (sección 6).

\section{Aproximación a la justicia a partir de la injusticia real}

La pregunta por la justicia siempre surge de hecho a partir de la experiencia dolorosa de su contrario, la injusticia, de modo que las aproximaciones más adecuadas a la justicia siempre surgen del mundo real del conflicto y de la injusticia. Por ello, para obtener un concepto de justicia, realmente adecuado a nuestro tiempo, conviene preguntamos sobre la realidad de la injusticia concreta, lo cual siempre conocemos mucho mejor.

Empecemos diciendo que la experiencia de la injuslicia que sufrèn los carnpesinos o las trabajadoras en el tercer mundo hoy bordea con la esclavitud. $\mathrm{Y}$ 
para ver esto con más claridad, analicemos los elementos fundamentales del ejet plo extremo de la esclavitud. En primer lugar, el esclavo vive en gran (has total) dependencio del amo sin que éste dependa de aquél, por lo menos sin q dependa de un esclavo en particular. Esto significa que el amo tiene poder sob su esclavo; es decir, puede tomar decisiones por él y así limitar su libertad. I segundo lugar, en esta situación, el esclavo no puede pedir cuentas al amo, y. se afiade a lo anterior, esto implica que al esclavo se le ha negado particip efectivamente en las formas reales de una relación que le afecta tan directame te. Por último, el amo ha reducido al esclavo a un objeto, aplastando su carácl real de sujeto, es decir, de persona capaz de tomar iniciativas y de actuar a libertad. Dada esta situación global de injusticia, el amo llega a cometer injus. cias concretas, obligando al esclavo a servir a fines contrarios a sus propi intereses.

El ejemplo de la esclavitud es extremo, ciertamente, pero es también ú para ilustrar lo que ocurre en la vida cotidiana. Muchos empleados ante sus jeft por ejemplo, algunos estudiantes ante sus profesores, inquilinas ante el casero campesinos arrendatarios ante el terrateniente, comparten, aunque en menor gr do, los elementos fundamentales que constimyen la siluación del esclavo.

Desde esta perspectiva podemos definir la injusticia de la siguiente manes es una relación social en la cual una persona o un grupo manliene a otros a situación de dependencio unilateral (no necesaria), es decir, ejerce poder sob ellos y les niega la capacidad de pedir cuentas.

Según esto, entendemos por injusticia, ante todo, una relación social, lo cu ilumina también lo esencial de la injusticia en el mundo real (el analogatu princeps, que dicen los filósofos). Desde la injusticia entendida como relaci social es fácil ampliar la definición de injusticia, de modo que abarque tambi los abusos concretos que reducen a objeto la realidad de la persona. $Y$ de es comprensión de la injusticia como relación social, se puede proceder fácilmen a la comprensión de la injusticia que se encama en instituciones, estructuras sistemas sociales, las cuales sostienen y sirven de base a las relaciones sociale Estas instituciones, estructuras y sistemas son injustos en la medida en que reduc a objetos a personas y grupos, mantienen dependencias innecesarias, limitan libertad, niegan la participación que puede exigir y pedir cuentas por el uso d poder y obligan a personas y grupos a servir a fines ajenos a sus propios intereses

Anadamos ahora tres reflexiones a esta definición de injusticia. La prime es que la dependencia, incluso la dependencia unilateral, no es en si misma injusi cuando llega a ser necesaria, como en el caso de niflos pequentos que depend de sus padres, o de personas incapaciadas que dependen de orros, o de un crimin cuya libertad se tiene que resiringir para proteger a los demás. Además, tod 
responsabilidades y pedirles cuenta por sus acciones. Según esto, la injusticia consiste en mantener a las personas en una dependencia unilateral e innecesaria, negándoles a la vez la posibilidad de pedir cuentas.

En segundo lugar, hay que advertir que con esta definición no se quiere negar en modo alguno la necesidad de autoridad en cualquier organización humana o el legítimo ejercicio del poder en general. Pero sí se trata de desenmascarar el abuso de la autoridad, es decir, el autoritarismo y el abuso de todo tipo de poder.

Digamos, por último, que lodos vivimos inmersos en situaciones injustas, aun cuando no carguemos necesariamente con la responsabilidad principal en dichas situaciones. Si en una sociedad pobre y marcada por la justicia, por ejemplo, alguien da empleo a una cocinera, ésta se encontrará, de hecho, en situación de dependencia unilateral. La situación es injusta, aunque no necesariamente por prácticas explícitamente injustas del empleador, sino por las estructuras de la sociedad. El empleador no tiene responsabilidad plena y directa del funcionamiento del mercado laboral y sus efectos, pero sí tiene obligación en justicia de humanizar la situación de la empleada, sobre todo dándole la posibilidad de que le pueda pedir cuentas.

Sea ésta nuestra primera conclusión: si entendemos lo que es la injusticia, comprenderemos fácilmente su contrario, la justicia. Si la injusticia consiste en una relación social de dependencia innecesaria y sin posibilidad de apelación, entonces hacer justicia será rectificar esa situación, y la justicia consistirá en que se den aquellas relaciones sociales donde prevalezca su contrario: la interdependencia, al menos en el sentido de la mulua capacidad de poder pedir cuentas eficazmente.

Introducir en el concepto de justicia la capacidad de pedir cuentas e insistir en ello refleja, sin duda, el ethos democrático de la modemidad. Sin embargo, este concepto de justicia no es sino una traducción actual de lo que la misma Biblia dice sobre la justicia.

\section{La justicia en la Biblia: Sedeq, Mishpat, Sedagah}

\subsection{Antiguo Testamento}

El concepto de justicia recorre todo el Antiguo Testamento. No se trata, sin embargo, de una justicia imparcial en el sentido occidental: la justicia conforme a la norma abstracta de "dar a cada uno lo suyo". En la Biblia, la justicia siempre se refiere más bien y en primer lugar a un contexto concreto de relaciones sociales. Especificamente, justicia significa rescatar a la víctima, liberar al oprimido. Expresa, por tanto, algún tipo de reivindicación'.

1. Sedeq (justicia) es expresión suprema y global de lo que es valioso, justo y correcto en la comunidad; es el Bien. Sedeq es el concepto central que gobier- 
na todas las relaciones sociales. Significa rectificar situaciones entre personas y grupos, vivir conforme a lo que la situación social exige. Significa, por tanto, justicia para el oprimido. ( $C f$. Jer 50,7 ; Is $41,2.10 ; 42,6 ; 45,8 ; 51,5$.)

Mishpat, sedagah, hesed-y-emeth (amor constante) y yeshuah (liberación, salvación) pertenecen al campo semántico de sedeq, justicia. Explicitan uno o más aspectos de sedeq o matizan el concepto. Ahora sólo vamos a explicar mishpat y sedagah, los cuales, después de sedeq, son los valores más importantes del Antiguo Testamento ${ }^{2}$. Pues bien, mishpat y sedaqah también tienen que ver con el cumplimiento de responsibilidades en relaciones sociales concretas.

2. Sedagah significa un acto de bondad o de compasión. En este sentido sedagah es liberar al oprimido, reivindicar al huérfano, a la viuda, al inmigrante, al pobre, contra sus opresores. En este sentido, el anuguo Cántico de Débora habla también de las sidqoth (plural de sedaqah) de Yahvé Dios (Jue 5, 11; cf. ISam 12, 7; Mi 6, 5; Sal 103, 6; Dan 9, 16; etc.), es decir, de "las acciones liberadoras de Dios". (Ez 18, sin embargo, emplea el término sedaqah en un sentido más amplio).

3. Mishpar se traduce con frecuencia por "derecho" o "justicia". Tiene matices jurídicos (regla, juicio, ley, proceso jurídico), pero éslos son sólo ampliaciones de su sentido primario: justicia liberadora, salvífica. De hecho, lo que está en el corazón de la wrah, ley de Israel, mishpat, consiste simple y sencillamente en hacer justicia, especialmente allá donde reina lo contrario. Mishpat significa, entonces, liberar al oprimido. "Busquen mishpar; den sus derechos al oprimido. Hagan justicia al huérfano; $y$ defiendan a la viuda" (Is 1,17 ).

Mishpat, además, está relacionado intrínsecamente con hesed (amor), rahamin (compasión) y sedaqah (acto de bondad). La Biblia no conoce, pues, ninguna justicia sin amor y sin misericordia para con la víctima. Contrariamente a la tradición occidental, en la Biblia no hay tensión entre justicia y misericordia. (Véanse Mi 6, 8; Os 12, 7; Zac 7, 9; Is 30, 18; Sal 119, 149.156).

En resumen, sedeq, mishpat y sedaqah significan "justicia", expresan los valores más importantes de la comunidad de Israel y constanternente aparecen juntos en la Biblia. Con distintos matices, comparten un núcleo común de significado: liberación de los oprimidos, auxilio para los necesitados.

\subsection{EI Nuevo Testamento}

Jesús proclama el reino de Dios que representa la realización de la justicia (sedeq y mishpat) de Dios (ML 5, 20;23, 23; cf. Lc 6, 20; etc.). De hecho, en lugar de "reino de Dios", Pablo habla de "la justicia de Dios" (Rm 1, 17; 3, 21; cf. 14, 17), que tiene un sentido no sólo social, sino personal y global: integridad. (Cf. también 2Pe 3,13.)

Más importante todavía es la constalación de que, según el Nuevo Testamen-

Digitalizado por Biblioteca "P. Florentino Idoate, S.J."

Universidad Centroamericana José Simeón Cañas 
to Jesús, especialmente en Mateo, realiza la justicia de Dios en su propia persona (Mt 5, 6; 12, 18-20; Rom 1, 17s; $c f .3,21 ; 5,18$ ). Su preocupación por los pobres y marginados (pecadores, mujeres, enfermos, samaritanos, niffos) encarna la justicia del Dios justo. Finalmente, Jesús muestra de manera explícita lo que quedaba implícito en el Antiguo Testamento: el amor al prójimo es la norma suprema del sedeq de Dios y resumen de todas las demás normas. El amor - tratar al prójimo como uno quiere ser tratado, como otro "yo" (Mt 7, 12) constituye la base y el alma de toda justicia: las normas y criterios de justicia tienen que expresar las exigencias del amor, los que siguen a Jesús en esta praxis se convierten en la justicia de Dios presente en el mundo (2Cor 5,21 , etc.). Jesús se convierte en el criterio último de lo que es la justicia: practicar la justicia es seguir a Jesús ${ }^{3}$.

\subsection{Conclusión}

La Biblia aporta elementos claves para un adecuado concepto de justicia, los cuales coinciden, en gran parte, con el análisis que hemos hecho más arriba. La Biblia, ante todo, ubica la pregunta por la justicia en un contexto realista de conflicto histórico. Preguntarse "qué es la justicia" significa preguntarse "cómo arreglar esta situación real de injusticia", 0 , al menos, "cómo responder a la necesidad del projimo". Esto significa, en segundo lugar, que la justicia consiste en rectificar relaciones sociales, pues las personas no son consideradas corno individuos aislados que exigen "lo suyo", como puede ocurrir en la tradición occidental. La Biblia, más bien, ve a las personas como inmersas en redes de interrelaciones sociales y plantea el hacer justicia como la tarea de rectificar esas relaciones dentro de la comunidad. (De hecho, el pensamiento clásico de occidente no suele olvidar este punto fundamental, pero sí lo olvida el liberalismo moderno, como veremos más adelante.) Por último, al proponer la justicia como defensa y liberación de las vícuimas, la Biblia la presenta como expresión del amor social, elemento que hay que añadir a la definición "minimalista" de justicia que hemos ofrecido en la primera sección.

La Biblia no aporta, pues, ni pretende aportar, todos los criterios necesarios para determinar si una situación o una práctica es justa o no, recordatorio especialmente importante en el mundo actual, tan complejo y tan distinto al de entonces. Sí lo ha intentado hacer el pensamiento occidental, que ha elaborado una larga y rica reflexión sobre la justicia, y puede, por ello, ayudar a elaborar criterios e instrumentos para dar luz sobre la situación actual, aunque, por otro lado, necesita ser criticado a la luz de lo dicho hasta ahora.

\section{La justicia en el pensamiento occidental}

En términos generales, para el pensamiento occidental la justicia significa dar a cada uno lo suyo. Históricamente, los griegos impulsaron la reflexión 
sobre la justicia, los romanos anadieron la perspectiva del derecho y el pensamiento modemo y contemporáneo ha matizado y criticado estas tradiciones.

\subsection{Los griegos: la justicia}

Platón y Aristóteles ofrecen dos aportes fundamentales de la filosofía griega clásica.

a) Para Platón justicia significa "hacer cada uno lo suyo":

Aquello que desde el principio, cuando fundábamos la ciudad, afirmábamos que habla que observar en toda circunstancia, eso mismo o una forma de eso es, a mi parecer, la justicia $Y$ lo que establecimos y repetimos muchas veces, si bien recuerdas, es que cada uno debe atender a una sola de las cosas de la ciudad: a aquello para lo que su naturaleza esté mejor dotada (República, n. 432).

Platón tiene, pues, un concepto funcional de la justicia, y según ello una sociedad puede ser llamada justa cuando cada parte de la sociedad (filósofos, militares, comerciantes, trabajadores, mujeres, etc.) cumple con su función. Y de modo semejante, una persona es justa cuando cada parte del alma (racional, irascible, concupiscente) cumple con su propia función.

AJ hablar de las virtudes "cardinales", Platón enumera cuatro: la prudencia (virud de filosofos y de la parte racional del alma), fortaleza (virud de militares y de la parte irascible), templanza (virud de trabajadores y de la parte concupiscente). La justicia es la que ordena el todo y consigue que cada parte de la sociedad y de la persona "haga lo suyo".

Este concepto de justicia, aunque ingenioso, da por supuesto que las personas desempeffan papeles sociales lijos, con lo cual se acepla la desigualdad y se propicia poco la libertad y la movilidad social.

b) Aristóteles afirma que la justicia es una virtud, es decir, un hábito o actitud firme, y comprende su contenido en el sentido general de integridad en las relaciones con los dernás. Pero, adernás, distingue dos formas específicas de justicia.

Justicia distributiva, es decir, lo que la comunidad debe a cada individuo o grupo, y para determinarlo hay que fijar criterios:

Todos eslán de acuerdo en que la distribución jusla debe determinarse en base a lo que cada cual merece. Pero no todos tendrín el mismo criterio de mérito: los demócratas dicen que es nacer libres, los oligarcas dicen que es la riqueza o nacer de una familia noble, y los aristócratas dicen que es la excelencia, o ser mejor (Etica a Nicómaco, V. 2, 1131a).

El mismo Aristóteles llegó a compartir muchos elementos de la visión aristocrálica. 
Justicia conmusativa, justicia "horizontal", es decir, lo que una persona o grupo debe a otra persona o grupo, como por ejemplo, cumplir contratos, pagar deudas, etc.

\subsection{Los romanos: el derecho}

Los romanos desarrollaron un amplio sistema jurídico y dieron un carácter marcadamente legal al concepto de justicia. Fue el jurista romano Ulpiano el que propuso la definición: Iustitia est constans et perpetua voluntas suum cuique iribuendi, es decir, la voluntad firme de dar a cada uno lo suyo. Platón ya habla rechazado este concepto de justicia, atribuyéndolo al pensador pre-socrático Simónides, pero la definición de Ulpiano llegó a prevalecer en el pensamiento occidental, especialmente después de que santo Tomás la adoptó como suya (S.Th. II-II, q. 58, a. 1).

\section{La filosofia moderna y contemporánea}

La sociedad tradicional deterninaba "lo suyo" en base a notables desigualdades y la lilosofía tradicional toleró extremos de desigualdad poco aceptables para la conciencia modema. Los antiguos creyeron que unos nacen para mandar y otros para obedecer, y no se cuestionaron las funciones tradicionales de la mujer, del obrero, elc., y tampoco la Biblia escapa del todo a estos presupuestos. A esto hay que afradir que el pensamiento tradicional ha enfatizado, en la práctica, la dimensión "romana", legal, de la justicia, contribuyendo con ello a la tendencia a pensarla en términos de respeto al orden -o desorden- establecido.

Como reacción a estas limitaciones, el pensamiento moderno y contemporáneo ha recalcado, por una parte, la igualdad y los derechos humanos, pero, por otra, ha vivido una "crisis" permanente: no ser capaz de fundamentar filosóficamente el concepto de justicia. A esto se debe la gran variedad de planteamientos que varnos a exponer a continuación muy brevemente.

a) Para el positivismo y el historicismo justicia es lo que decide cada comunidad: lo moral es igual a lo legal. Esta corriente no supera el legalismo y no posee criterios objelivos para criticar las estructuras y leyes vigentes.

b) El kantismo exige que el trato humano se haga según normas racionales, es decir, un trato igual y universal. Se eliminan los privilegios y hay que dar una justificación para el trato especial. No se trata a las personas como puros medios.

c) El utilitarismo se fija en las consecuencias reales de las acciones, no en reglas abstractas, de ahf que justicia signifique realizar el mayor bien para el mayor número de personas. Este concepto de justicia, sin embargo, liende a restar importancia a valores que son poco cuantificables y corre el riesgo de sacrificar, en nombre de la mayoria, derechos de individuos o de grupos minorilarios. 
d) El marrismo casi nunca usa la palabra justicia. Implícita en el marxismo, sin embargo, está una idea de justicia como "apropiación" de lo humano. Se supone que bajo condiciones pre-socialistas los seres humanos se han "alienado" -o separado - de lo suyo: el trabajo y su producto, los semejantes, la naturaleza, etc. Marx establece también un criterio: "de cada uno según su capacidad, a cada uno según su necesidad" (Crítica del Programa Gotha).

e) Para el contractualismo, justicia es lo que los miembros de la sociedad establecen, explícita o implícitamente, por contrato, y la diferencia con respeto al positivismo consiste en que aquí se define un procedimiento "justo" para ese contrato. Detengámonos a analizar este planteamiento un poco más en detalle porque las recientes formas de contractualismo son las que más han estimulado la reflexión sobre la justicia en los últimos veinte años. Las figuras principales son John Rawls ("la justicia como imparcialidad" Uairness])" y Jürgen Habermas ("la ética del discurso")s. Ambos intentan unificar las intuiciones de Kant con la tradición del contrato social.

Rawls argumenta que los criterios para determinar qué es justicia deben ser establecidos de la siguiente manera. Supóngase que los miembros de la sociedad se encuentran en una "posición original" planilicando los criterios e instituciones que van a gobernar su vida social. Supóngase, también, que planifican imparcialmente, tras un "velo de ignorancia", sin saber ninguno de ellos sus propias capacidades o la posición social en que se puedan encontrar en esta sociedad. Bajo estas condiciones, argumenta Rawls, los participantes maximizarian la libertad para lodos y permitirían la desigualdad sólo si ésta prometiera beneliciar a todos y, especialmente, al peor situado en la sociedad. Con este último criterio se intenta superar el peligro de uúlitarismo que puede sacrificar a algunos para beneficiar a la mayoriá.

Para Habermas, este ejercicio ficticio de Rawls no basta para definir la justicia. Esta viene definida, más bien, por las normas que resultan, o pueden resultar, de un discurso real sobre las mismas, discurso abierto en principio a todos?. Es decir, la justicia es el contenido de aquellas normas cuyas consecuencias resultarían aceptables a todas las personas a quienes afecten las normas".

Tanto Rawls como Habermas ofrecen importantes aportes para superar las dificultades en que ha caído el pensamiento moderno. Sin embargo, es evidente que ambos conceptos de justicia se refieren meramente al procedimiento, son, pues, procedimentales, es decir, formales. Señalan el proceso que conduce a fijar el contenido de la justicia, pero no fijan prácticamente ningun contenido de ella. En definitva, aceptan un positivismo de contenidos.

Llama la atención, además, que el procedimiento presupone la posibilidad imagirativa de determinar la vida social ("posición original" de Rawls) o la posibilidad real de unir a personas con intereses distintos (como por ejemplo empresarios y obreros) para dialogar y hasta concertar en la práclica. Pero es muy

Digitalizado por Biblioteca "P. Florentino Idoate, S.J."

Universidad Centroamericana José Simeón Cañas 
claro que la situación real de las grandes mayorías de América Latina - las victimas de la injusticia - se caracterizan por todo lo contrario, es decir, por la imposibilidad de exigir y pedir cuentas a los que si planifican y deciden. Y se caracteriza, además, y muy específicamente, por lo que es el paso previo para determinar el procedimento: la exclusión de las mayorías de la mesa del discurso' y, consecuentemente, la falta de concertación social.

Esto implica que, aunque los aportes de Habermas y Rawls puedan servir de ideales y estúmulos utópicos para América Latina, no logran historizar el concepto de justicia para la acual realidad latinoamericana. Esa historización tiene que pasar, por necesidad, por la experiencia de dependencia unilateral y la falta de capacidad de pedir cuentas, que es el pan de cada día de las grandes mayorías del continente. Y esta experiencia negativa apunta ya a ciertos contenidos de la justicia: aquellas relaciones sociales donde prevalezca la interdependencia, en el sentido (mínimo) de la capacidad mutua de pedir cuentas eficazmente, de una u otra manera.

\subsection{Conclusión}

Este breve recorrido por la histria del pensamiento occidental sobre la justicia muestra que, a pesar de su riqueza, las nociones tradicionales de griegos y romanos aceptan desigualdades intolerables. En este sentido coincidimos con el pensamiento moderno cuando afirma que la justicia tiene que cuestionar el (des)orden establecido a la luz de la igualdad humana y la libertad y, en general, de los derechos humanos. Pero, a su vez, la ética moderna carece de un fundamento sólido y suele caer en el individualismo. Insiste mucho en los derechos personales y poco en las obligaciones para con la comunidad. Sollo con dificultad especifica el contenido de la justicia en términos de las condiciones sociales necesarias para una vida realmente humana.

Ante estas limitaciones de la tradición occidental pensamos, con la Biblia, que en situaciones reales de injusticia la justicia no es sólo dar a cada uno lo suyo, sino que es restaurar lo despojado $0^{10}$. Sin embargo, es claro también que la Biblia no lo dice todo para el mundo actual, y que, por otro lado, aun con sus limitaciones, tanto la ética tradicional como la ética modema tienen algo que ofrecer. De ahí que para historizar en lo concreto el concepto de justicia es necesario integrar el pensamiento secular en la perspectiva blblica, como de hecho lo ha intentado siempre -en mayor o menor fidelidad a la Biblia- la teologia católica.

\section{La tradición católica ${ }^{11}$}

En los primeros siglos del cristianismo la teología llevó a cabo una cierta integración de perspectivas blblicas y grecorromanas sobre la justicia. Sin embargo, la edad media perdió poco a poco la originalidad de esta integración

Digitalizado por Biblioteca "P. Florentino Idoate, S.J."

Universidad Centroamericana José Simeón Cañas 
en razón de la progesiva divergencia entre fe y vida, ascética y ética, contemplación y acción, para terminar en la época postridentina en la total reducción de la justicia al derecho natural. El propio magisterio social de la Iglesia hasta Juan XXIII no se aparta de una concepción ético-jurídica de la justicia. Habrá que esperar al concilio Vaticano II y la teología de su entomo -en particular al sínodo de obispos de 1971 sobre "la justicia en el mundo"...- para recuperar la originalidad bíblica y propiamente cristiana, fuente de una écica teológica y de una espiritualidad de la justicia ${ }^{12}$.

A partir de la edad media la tradición cristiana ha distinguido tres uipos de justicia: la justicia distributiva, la justicia conmutativa (que se remontan, ambas, a Aristóteles) y la justicia social. Cada una de estas formas de justicia corresponde a un tipo de relaciones sociales. Como hemos visto, también la Biblia concibe la justicia precisamente en términos de relaciones sociales, y por ello la triple división clásica debiera ofrecer posibilidades para precisar y concretar intuiciones bíblicas. Por un lado, la distinción tripartita ha demostrado un gran poder iluminador; pero, por otro, históricamente, muchas interpretaciones de este esquema han quedado demasiado aferradas a presupuestos jerárquicos y autoritarios de las sociedad tradicional. Afortunadamente, en los últimos affos se ha ido repensado la diferenciación de los tres tipos de justicia ${ }^{13}$, con una interpretación que puede ser muy útil para historizar hoy la noción bỉblica de justicia. Veámoslo.

La justicia distributiva exige que lodos tengan acceso a los medios necesarios para satisfacer sus necesidades básicas (por ejemplo, el trabajo, la educación, la propiedad, la salud) y que nadie sea excluido de los productos básicos de la sociedad. Como afirma la carta de los obispos católicos de Estados Unidos Justicia económica para todos (1986) ${ }^{14}$ - de la que citaremos algunos párafos-, "la justicia distributiva exige que se evalúe la distribución de los ingresos, las riquezas y el poder en la sociedad a la luz de su impacio sobre las personas cuyas necesidades materiales básicas quedan sin satisfacer" (n. 70).

La justicia conmutativa "exige que todos los convenios e intercambios entre individuos o grupos sociales privados se hagan equitativa y honradamente" (n. 69), exige fidelidad a contratos, acuerdos e intercambios, y que quienes suscriben esos acuerdos sean partes libres y lundamentalmente iguales.

Justicia social es un término técnico que tiene un significado distinto al que liene en el uso corriente de la expresión. La justicia social en el sentido de la carna exige que cada ciudadano conuribuya al bien común, es decir, a la construcción y mantenimiento de una comunidad que ofrezca una vida realmente humana para sus miembros. Exige también que la comunidad brinde condiciones para que todos puedan contribuir a esa sociedad. "Según la justicia social, las personas deben participar activa y productivamente en la vida de la sociedad, en tanto que la sociedad liene el deber de crear las condiciones para que esa participación sea posible" (n. 71).

Digitalizado por Biblioteca "P. Florentino Idoate, S.J."

Universidad Centroamericana José Simeón Cañas 
Estas tres dimensiones de la juslicia deben interrelacionarse y matizarse mutuamente, y de esta forma, además, se evitan enfoques unilaterales. Por ejemplo, la patronal puede afirmar que el contrato laboral es justo por haber sido firmado por las dos partes. Pero esto no quita la posiblidad de que sea injusto por falta de justicia distributiva, es decir, porque las desigualdades son tan grandes que los obreros están ante la alternativa de tener que aceptar el contrato o morir de hambre. En ese caso, es obvio que no entran libremente al contrato, sino por la Iuerza.

La justicia triparlita critica, pues, las grandes desigualdades sociales sin caer en el individualismo, y ayuda a elaborar criterios como los siguientes:

La justicia fundamencal exige que se establezcan niveles minimos de participación en la vida de la comunidad para todas las personas (n. 77).

El deber de conseguir justicia para lodos significa que la reivindicación económica más urgente para la conciencia de la nación es la de los pobres (n. 86). La satisfacción de las necesidades básicas de los pobres es de la más alta prioridad (n. 90).

Una prioridad muy importante es incrementar la participación activa en la vida económica de quienes aclualmente son excluidos o son vulnerables (n. 91).

Se debe orientar la inversión de la riqueza, el talento y la energía humana hacia el beneficio de los pobres y los económicamente inestables (n. 92).

Se deben evaluar continuamente las políticas económicas y sociales, y lambién la manera de organizar el mundo del trabajo a la luz de su impacto sobre la fuerza y la estabilidad de la vida familiar (n. 93).

El Papa Juan Pablo II [declaró] ... que "las necesidades de los pobres son de mayor prioridad que los deseos de los ricos; los derechos de los obreros son de mayor prioridad que el maximizar las ganancias; la conservación del medio ambiente es de mayor prioridad que la producción con fines militares" (n. 94).

Es evidente que estos criterios que hemos mencionado reflejan un esfuerzo serio por integrar en la tradición occidental la preocupación biblica por los pobres y débiles. Más todavía, Justicio económica para todos interpreta la justicia en términos de participación. Hay que repartir, no sólo los bienes, sino también el poder. En esto, la carta pastoral coincide con el consepto de justicia que hemos desarrollado en la sección 1 y con la ética de la liberación en general.

\section{Etica de la liberación}

Durante las últimas décadas en América Latina se ha desarrollado una ética de la liberación ${ }^{13}$. Como la Biblia, parte de la injusticia y el conflicto en la 
historia. Plantea la justicia en términos de liberación de la opresión y de la dependencia, por un lado, y de llegar a la paricipación y la comunión, por otro. A esta corriente de pensamiento pertenece lo que dijimos en la primera sección, por lo cual no nos vamos a extender ahora en su análisis. Digamos, resumiendo, que justicia significa liberación de una situación de dependencia unilateral y en la que tampoco se da la capacidad de pedir cuentas, y significa liberación para llegar a una siluación de interdependencia con capacidad de pedir cuentas por el uso del poder, a lo que hay que agregar, según el Nuevo Testamento, que la justicia sólo puede vivir y mantenerse cuando se entiende y se practica como obra del amor.

\section{Una aplicación concreta: evaluación del capitalismo y del socialismo}

En este apartado final queremos aplicar el concepto de justicia que hemos elaborado a la evaluación de los sistemas económicos actuales. Al hacerlo, somos bien conscientes de lo que advierte un gran economista. Al diseñar un sistema social para las sociedades modemas, decía, se trata de "un problema grande y de cerebros pequeños". Esto, sin embargo, no nos debe paralizar.

Con relación a los sistemas económicos modernos debemos preguntarnos, ante todo, dónde está el meollo de la cuestión moral. Y para responder debemos fijamos en dos criterios. El primero es cómo afecta un sistema (o una medida concreta) a los pobres y débiles. Y el segundo es si el pueblo puede pedir cuentas por el uso que se hace de los medios de producción. El primer criterio se fija en los efectos, el segundo apunta a las causas.

El primer criterio básico consiste en analizar el impacto que tiene la economía (o un programa económico) en la vida de los pobres. El sistema económico tiene que satisfacer las necesidades básicas de las mayorias ${ }^{16}$, y por ello una economía política o un programa -de ajuste estructural, por ejemplo- es inmoral en la medida en que atenta contra la vida de las mayorías. En América Latina el hecho es claro: la política económica de los países latinoamericanos atenta en gran medida contra la vida de las grandes mayorias. De ahí que hay que denunciar como inmorales programas de ajuste estructural -y otros semejantes- en cuanto recaen en forma desproporcionada sobre los hombros de los pobres.

El segundo criterio básico es estructural. Una ética social tiene que ir más allá de los efectos observados y preguntarse cuáles son las causas estructurales que están en la raíz de los constantes abusos. Captar los efectos negativos es importante para la denuncia, pero sólo llegando a las raices del problema se podrá diagnosticar y proponer altemativas humanas factibles. Este criterio básico estructural consiste en la capacidad que tienen o no tienen los afectados para pedir cuentas a los que adminstran la propiedad productiva. Veámoslo.

En el mundo industrializado hay dos factores que determinan el carácter de un sistema social: quién controla la propiedad productiva y cómo funciona el

Digitalizado por Biblioteca "P. Florentino Idoate, S.J."

Universidad Centroamericana José Simeón Cañas 
mercado (con mayor o menor intervención del gobierno). Bajo el capitalismo, los propietarios particulares son duefios de la propiedad productiva y favorecen el funcionamiento libre del mercado (oferta y demanda), y, además, el Estado tiende a intervenir en la economía para servir al capital. Bajo el socialismo, el Estado administra la propiedad productiva; busca controlar precios y salarios, y hasta eliminar el mercado como mecanismo regulador de la economía. En ambos casos, la postura ante el capital y el mercado son factores determinantes para la configuración de la sociedad.

Para evaluar éstas u otras políticas económicas son varios los criterios "estucturales" que se pueden utilizar: la posición del trabajador, el papel del Estado y del mercado, el grado de propiedad privada, etc. Pero el más decisivo parece ser el manejo de la propiedad productiva, pues la industrialización y la modemización, en general, tienden a crear unidades productivas cada vez más grandes y poderosas, como los bancos, las fábricas, etc. Entonces, controlar los medios de producción (ahora, especialmente, las finanzas y la tecnología) significa controlar la economía y toda la sociedad. En el capilalismo liberal extremo, una élite de ricos controla la sociedad; en el comunismo colectivista extremo, los burócratas del gobiemo (o el partido) controlan la sociedad. En el primer caso, los trabajadores sirven al capitalista; en el segundo, sirven al aparato del Estado. En todo caso, la vida de los trabajadores y de sus familias depende decisivamente de los administradores de la propiedad productiva, y a ellos con frecuencia se les niega lo necesario para una vida digna.

En esta siluación, no se trata, por supuesto, de argumentar en favor de un centrismo aristotélico, sino de constatar que los capitalismos reales y el socialismo real del bloque del Este han padecido del mismo defecto estructural: los administradores de la propiedad productiva no tienen que rendir cuentas por el manejo de la misma. Este delecto se manifiesta concretamente en la falta de participación real de parte de los trabajadores en la gestión de la empresa Los trabajadores no se sienten trabajando "en algo suyo" (Laborem exercens 15). La empresa no es "una auténtica comunidad humana" (Mater et magistra 91). Y si el urabajo no es un proyecto común, entonces tampoco lo es la economía. Y, por eso, la sociedad misma se divide en trabajadores por un lado y élite por otro.

Este es el núcleo del problema moral de las economías modernas, sean éstas capitalistas o socialistas. El problema no consiste, por lo tanto, en que un pequeño grupo de expertos administre la economia o el capital, lo cual es prácticamente inevitable en la economía modema. La gran pregunta es si estos administradores tienen que rendir cuentas ante el pueblo por el manejo de los medios de producción. ¿Puede el pueblo pedir cuentas por el uso de los recursos de los que depende para vivir, sí o no? ¿Hay democracia económica, sí o no?

La actual coyuntura no se presta a soluciones globales, pero a la luz de lo anterior, podriamos formular, para el mediano plazo, más allá de la protesta, la

Digitalizado por Biblioteca "P. Florentino Idoate, S.J."

Universidad Centroamericana José Simeón Cañas 
siguiente tarea concreta para el movimiento laboral y para una Iglesia profética: insistir en la capacidad que deben tener obreros y campesinos de pedir cuentas a la patronal ( $y$, por supuesto, a las cooperativas). Eso debe hacerse en forma de participación real en la gestión de la empresa y de la economía a todos los niveles, y en el reparto de beneficios, ambos criterios importantes en la doctrina social oficial, pero que tienen poca resonancia en la práctica ecclesial ${ }^{17}$.

\section{Conclusión}

"Hacer justicia" (como acción) significa superar la injusticia histórica, es decir, superar la dependencia unilateral donde los afectados no pueden pedir cuentas a los detentadores del poder. Y "la justicia" (como situación) significa la existencia de relaciones de interdependencia y la capacidad de pedir cuentas.

La insistencia en esto último surge del ethos modemo democrático, pero recupera elementos fundamentales de la justicia en la Biblia y ayuda a superar muchas dificultades provenientes de la tradición occidental. Aunque la doctrina social católica no insiste en la capacidad de "pedir cuentas", sí hay en ella algunos elementos clave que apuntan hacia ello. En cualquier caso, este concepto de juslicia nos parece esencial para la necesaria historización de la justicia en el mundo de hoy.

\section{Notas}

1. Para el material de esta sección, $c f$. G. Von Rad, Teologra del Antiguo Testamento (Salamanca 1982), vol. I, 453-68; E. Berkovits, Man and God (Detroil 1969); Gotulob Schrenk, "dikaiosune", Theological Dictionary of the New Testament, G. Kittel, ed. (Grand Rapids), s.v.; Klaus Berger, "Justicia: I. Concepto de justicia en la Escritura", Sacramentum Mundi (Barcelona 1973), vol. 4, cols. 162-69; José Porfirio Miranda, Marx y la Biblia (Salannanca 1972), capítulos 2-4; J. P. M. Walsh, SJ., The Mighry from Their Thrones: Power in the Biblical Tradition (Filadelphia 1987), cap. 1-2; R. Aguire y F. J. Vitoria Commenzana, "Justicia", en Mysteriwn Liberationis. I. Ellacuría y J. Sobrino, eds. (San Salvador 1991), vol. II, pp. 539-61.

2. Sería difícil sobreestimar las implicaciones del comentario del estudioso judío Eliezer Berkovits: "Se puede dudar de que, entre las muchas ideas de la Biblia hebrea, exista alguna que haya sufrido más por equivocación y, con frecuencia, por falsificacion prejuiciada, que las de sedeq, mishpat, y sedagah". Man and God 340.

3. Nótese el paralelo con la tésis de Aristóteles, cuando afima que la medida del valor moral es la persona justa. Etica nicomaquea, XX. 4, 1166a12-13; X. 6, 1176b25-28; ete.

4. John Rawls, A Theory of Justice (Cambridge 1971); traduccion en español, Teoria de la justicia (México 1979).

5. Jürgen Habermas, Moralbewusstsein und kommunicatives Handeln (Frankfurt 1983); traducción en español, Conciencia moral y asción comunicativa, por Ramón García Cotarelo (Barcelona 1985). Karl-Otto Apel ha colaborado también, desarro- 
llando esta noción de la élica discursiva Véase K.O. Apel, "La étiea del discurso como ética de la responsabilided. Una transformación postmetafisica de la élica de Kent", en K. O. Apel, Teorla de la verdad y ética del discurso (Barcelona 1991) 147-184.

6. Primero, el posnulado de la libertad igual reza así: "Cada persona debe tener igual derecho al sistema total más amplio de libertades iguales básicas que son compatibles con un sistema similar de libertades para todos" ( $A$ Theory 302). Luego viene el postulado de difetencias: "Las desigualdades sociales y económicas, por ejemplo las desigualdades en riqueza y en autoridad, son justas únicamente si resultan en beneficios compensatorios para todos, y, en particular, para los miembros en la situación menos ventajosa de la sociedad" (ibid. 14s).

7. "De conformidad con la ética discursiva una norma únicamente puede aspirar a tener validez cuando todas las personas a las que afecta consiguen ponerse de acuerdo en cuanto participantes de un discurso práctico (o pueden ponerse de acuerdo) en que dicha norma es válida" (Conciencia moral 86). Habermas llama a este principio de la ética discursiva, el principio (D).

8. Este es el pastulado de la universalidad (U): "Cada nomna válida habrá de satisfacer la condición de que las consecuencias y efectos secundarios que se siguen de su ecatamiento general para la satisfacción de los intereses de cada persona (presumiblemente) puedan resultas aceptados por todos los afectados (así como preferidos a los efectos de las posibilidades sustitutivas de regulación)" (Jbid. 85s). Esta preocupación por las consecuencias, tanco en Habermas como en Rawls, indica una nuptura con el rigorismo kantiano que no toma en cuenta las consecuencias de los principios.

9. Cf. Enrique Dussel, "De la 'pragmática trascendental' a una 'económica trascendental'. Reflexiones desde la perspectiva de una élica de la liberación latinoamericana", en Etica en América Latina (Bogota 1991) 45-80. Aceptando la orientación bésica de la étiea discursiva, Dussel propone reinlerpretarla desde "el Otro" excluido.

10. Ver M. Vidal Moral de actitudes, Tomo III, 112-14. Para una presentación excelente de conceptos modemos de la justicia, tanto filosólicos como teológicos, véase Karen Lebacqz, Six Theories of Justice: Perspectives from Philosophical and Theological Ethics (Minneapolis 1986).

11. M. Cozzoli, "Justicia" en Nuevo diccionario de teologia moral, F. Compagnioni et al., eds. (Madrid 1992), s.v.; José Ignacio González Faus, "Justicia" en Conceptos fundamensales de pasioral, Casiano Floristán y Juan José Tamayo eds. (Madrid 1983), s.v.; R. Aguirre y F. J. Vitoria C., "Justicia" (nota n. 1 de este artículo); J. M. Díez Alegría, "Justicia: III. El concepto de justicia en filosofía y en teología moral" ел Sacramensum mundi, (Barcelona 1973), vol. IV, cols. 169-77; David Hollenbach, Claims in Conflict: Retrieving and Renewing the Catholic Human Rights Tradition (New York 1979), cap. 4; idem, Justice, Peace and Human Rights: American Catholic Social Ethics in a Pluralistic Context (New York 1988), caps. 2 y 5.

12. M. Cozzoli, "Juslicia" 984

13. Véanse, especialmente, los arúculos de Cozzoli y de Hollenbach señalados en la nota anterior. Está en juego aqui la relación entre las tres formas de justicia, especialmente la interpretación de la justicia "general" o "legal" y la superación del volutarismo de la escolástica tardía.

14. Conferencia Nacional Católica de Obispos, Justicia económica para todos: Carta

Digitalizado por Biblioteca "P. Florentino Idoate, S.J."

Universidad Centroamericana José Simeón Cañas 
pastoral sobre la enseñanza social casolica y la economia de Estados Unidos de America (Weshington 1987).

15. Cf. E. Dussel, Para una ética de la liberación latinamericana, tomos I y II (Buenos Aires 1973); idem, Filosofla éfica latinoamericana, tomos 3, 4 y 5 (México 1977; Bogotó 1979 y 1980). Para la érica teológica, cf. E. Dussel, Etica comunita. ria (Madrid 1986) y las referencias en Francisco Moreno Rejó, "Etica de liberación" en Conceptos fundamentales de érica teolbgica, M. Vida. ed. (Madrid 1992) 253-68.

16. Gaudium et spes, 63; Laborem exercens, 18-19.

17. Quadragesimo anno, 65; Mater et magistra, 32, 75, 91-92; Gaudiun et spes, 68; Medellín, "Justicia", 11-12; Laborem exercens, 14-15. 